https://doi.org/10.31713/m1118

\title{
DISINTEGRATION OF ORE-BEARING ROCKS AND ITS EFFECT ON THE CONTENT OF CONSTITUENT COMPONENTS
}

\section{Toktosunova B.B.}

Kyrgyz State University of Geology, Mining and Natural Resources Exploitation named after Academician U.Asanaliev, $\mathrm{PhD}$, Professor, Head of the Department of "Natural Sciences" of the Geological Exploration Faculty, Kyrgyz Republic

\section{Abdibaitov Sh.A.}

Kyrgyz State University of Geology, Mining and Natural Resources Exploitation named after Academician U.Asanaliev, Candidate of technical sciences, Associate professor of the Department of UMMD Faculty of Mining and Metallurgy, Kyrgyz Republic

\section{Kushnazarova S.Z.}

State Enterprise "Kyrgyzgeologiya", Directorate "Hydrogeologiya" in the position of "Hydrogeologist", Kyrgyz Republic

Tutasheva A. Z.

Kyrgyz State University of Geology, Mining and Natural Resources Exploitation named after Academician U.Asanaliev, student gr. D-117, Mining and Metallurgical Faculty, Kyrgyz Republic Kozhogulov M.B.

Kyrgyz State University of Geology, Mining and Natural Resources Exploitation named after Academician U.Asanaliev, student gr. AG (G) -1-20, the Geological Exploration Faculty, Kyrgyz Republic

\section{Annotation}

In this paper we have studied, analyzed, summarized scientific information on the black-shale formation of the Saryjaz area, determined the chemical composition of initial samples, and ore-bearing minerals broken down into different sizes and evaluated their constituent components. Separated magnetically susceptible from magnetically unsusceptible components of raw materials brought from the three points of the coordinates of the deposit and derived their percentage ratio. The size of dispersed particles was determined on a scanning electron microscope (SEM), 
and the morphological structure of the mineral and the influence of the size of dispersed particles on the enrichment of useful components was studied by it.

The purpose of the work: Study of the influence of the size of dispersed particles on the manifestation of useful components in the composition of ore-bearing rocks of the Saryjaz area black shale formation.

The influence of dispersion of particles on the increase in the quantitative content of useful components in the composition of ore minerals was established. It is shown that in a finely divided sample, the yield of the quantitative content of noble metals is several times higher compared to the control one.

Keywords: ore, crushing, black shale formation, Saryjaz area, minerals, deposits, mineral reserves, research methods, size of dispersed particles, spectral and elemental analysis, scanning electron microscope, point coordinates.

\section{Introduction}

It is known that ores are complex, polymetallic natural formations. Joint presence of several valuable minerals in ores makes it difficult to use them in metallurgical processes without preliminary separation by enrichment methods. There is a number of opinions of experts that the scale of use of minerals is continuously increasing, and the quality of ores is systematically worsening; the content of useful components in ores is decreasing. In recent years with the development of modern technologies and techniques of enrichment allows to involve in processing of all new types of minerals and requires the use of the most advanced technological processes and methods of analysis.

It is known the method of enrichment of minerals, including crushing the ore, separation of minerals of higher density from the crushed ore and removal of the waste rock and minerals of light fractions to the dump.

The method of decryption (this is the property of individual minerals to crack when heated rapidly and to collapse when cooled) is widely used in mineral processing [1].The way of application of nanotechnology [2] in enrichment and extraction from fine-grained ores of "black-shale" type was considered.

The processes of physical-chemical and bacterial [3] in the processing of refractory ores, technogenic combined methods of cuvette and heap leaching of gold (Patent 2350665) were noted. The bacterial method [4] of leaching intensification makes use of the ability of autotrophic bacteria (Thiobacillus ferrooxidans, Thio-bacillus thiooxidans and others) to absorb the energy released for their vital activity 
during the oxidation of sulfides and thiosulfates of metals and sulfur as well as during the transition of $\mathrm{Fe}^{2+}$ into $\mathrm{Fe}^{3+}$.

Despite the existence of numerous methods, the search for new environmentally friendly, more effective and economically profitable ways of enrichment of useful minerals as well as the desire to optimize the process of extraction remains a topical problem.

Therefore, in this work we conduct research on the development of optimal parameters in the process of enrichment of valuable components of ore minerals of black-shale formation of the Saryjaz area.

\section{Preparation of source materials}

It is known that the preparatory processes include crushing, grinding operations, aimed at the disclosure of valuable components of ore-bearing rocks and bringing the material to the size required in the process of enrichment.

The object of the study is the ore minerals of the black shale formation of the Saryjaz area brought from three points of the deposits by coordinates

Table

\begin{tabular}{|c|c|c}
\multicolumn{3}{|c}{ Coordinates of points } \\
Point 1. BSSF (a) & Point 2. BSSF (b) & Point 3. BSSF (c) \\
\hline$x-14344641$ & $x-14344943$ & $x-14337830$ \\
\hline$y-4678073$ & $y-4679611$ & $y-4683314$ \\
\hline$h-2731$ & $h-2738$ & $h-2927$ \\
\hline
\end{tabular}

Table 2

Geographic coordinates

\begin{tabular}{c|c|c}
\hline Point 1. BSSF (a) & Point 2. BSSF (b) & Point 3. BSSF (c) \\
\hline$x-42^{\circ}, 13^{\prime}, 19.6^{\prime}$ & $x-42^{\circ}, 14^{\prime}, 9.6 ”$ & $x-42^{\circ}, 16 ', 4.4^{\prime}$ \\
\hline$y-79^{\circ}, 7^{\prime} .4 .3^{\prime \prime}$ & $y-79^{\circ}, 7^{\prime} .16^{\prime \prime}$ & $y-79^{\circ}, 2 ' .2 .2^{\prime \prime}$ \\
\hline
\end{tabular}



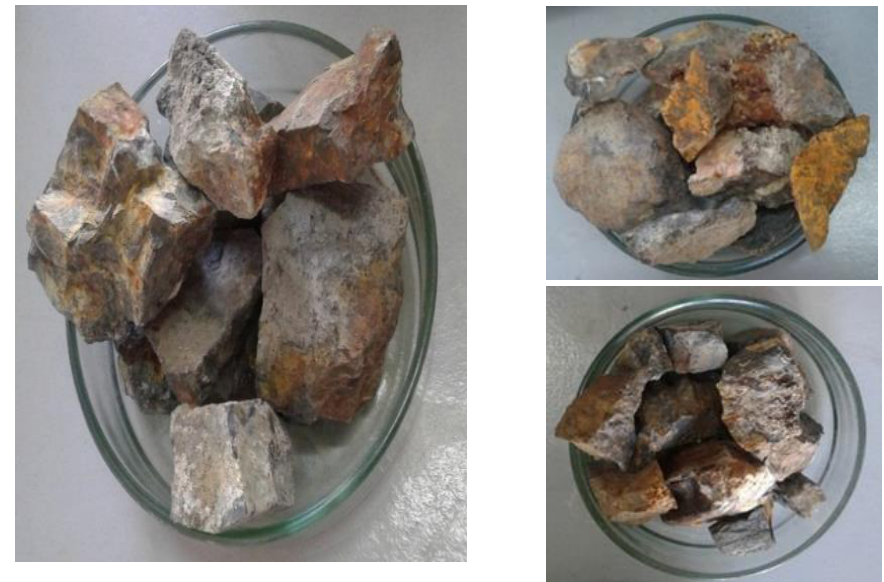

Fig. 1. Pieces of mineral brought from the deposits of the black shale formation of the Saryjaz area

At crushing used a mechanical method of crushing minerals, which was carried out in a crushing machine with a particle size (fine) $-10-15 \mathrm{~mm}$. For this purpose, the initial material weighed on scales of $20 \mathrm{~g}$ was taken. Further crushing is called grinding, which are used for ore going to enrichment. Then the crushed products obtained were sifted out on sieves (with three nearby mesh sizes) and each fraction was weighed separately.

At the same time, the duration and degree of crushing (grinding), power consumption (from the beginning to the end according to indications of the electric meter). The obtained data are presented in Table 3 .

Table 3

Ore crushing indicators

\begin{tabular}{l|l|c}
\hline № & \multicolumn{1}{|c|}{ Shredding indicators } & ore \\
\hline \multirow{2}{*}{1} & Size, mm: & $10-15$ \\
\cline { 2 - 3 } & Initial & $10-20 \mathrm{~g}$. \\
\cline { 2 - 3 } & intermediate & $15 \mathrm{~g}$. \\
\cline { 2 - 3 } & final & $5 \mathrm{~g}$. \\
\hline 2 & Crushing time, $\min$ & $30-60$ \\
\hline 3 & Crushing ratio, $i=$ Dmax $/ \mathrm{dmax}$ & $1,5-2 \mathrm{~mm}$ \\
\hline 4 & Specific power consumption, W/kg & 3-phase electric shift.3000 rpm, \\
& & $2 \mathrm{~kW}, 30-60 \mathrm{~atm} / \mathrm{cm}^{2}$ \\
\hline
\end{tabular}


Hence " $i$ " is the ratio of the diameter of the maximum piece of the source material $D_{\max }$ to the diameter of the maximum piece of the crushing or grinding product $d_{\text {max }}$.

With the method of separating magnetically receptive parts from magnetically non-receptive parts: it is observed that magnetically non-receptive parts amounted to $88.4 \%$; magnetically receptive $9.4 \%$; loss of $2.2 \%$ (Table 4 ).

Table 4

Results of separation of magnetically receptive parts from magnetically nonreceptive parts

\begin{tabular}{c|c|c|c|c|c|c|c}
\hline $\begin{array}{c}\text { Sample } \\
\mathrm{s}\end{array}$ & $\begin{array}{c}\text { Weight } \\
\text { of the } \\
\text { load } \\
\mathrm{c}, \mathrm{g}\end{array}$ & $\begin{array}{c}\text { magnetical- } \\
\text { ly non- } \\
\text { receptive } \\
\text { parts }\end{array}$ & $v, \%$ & $\begin{array}{c}\text { magnetical- } \\
\text { ly receptive } \\
\text { parts }\end{array}$ & $\begin{array}{c}\nu, \\
\%\end{array}$ & Loss & $v, \%$ \\
\hline $\begin{array}{c}\text { BSSF } \\
\text { (a) })\end{array}$ & $\begin{array}{c}24,4399 \\
0\end{array}$ & 21,50177 & 87,9 & 2,40100 & 9,8 & $\begin{array}{c}0,5371 \\
3\end{array}$ & 2,2 \\
\hline $\begin{array}{c}\text { BSSF } \\
\text { (b) }\end{array}$ & $\begin{array}{c}19,6845 \\
5\end{array}$ & 39,76 & 88,3 & 1,795 & 9,8 & 0,492 & 2,49 \\
\hline $\begin{array}{c}\text { BSSF } \\
\text { (c) }\end{array}$ & $\begin{array}{c}23,4779 \\
0\end{array}$ & 20,9504 & 89,0 & 2,0680 & 8,8 & 0,4595 & 2,0 \\
\hline Total: & & & 88,4 & & 9,4 & & 2,2 \\
\hline & & & & & & & $\begin{array}{c}100 \\
\%\end{array}$ \\
\hline
\end{tabular}

The study of the material composition of ore minerals consists of its two components: the determination of chemical (elemental) and mineral composition. Lumps of large size brought from the three points of the deposits were subjected to crushing to sizes $1.5-2 \mathrm{~mm}$ in a ball mill held screening $[5,6,7]$, then determined their chemical composition, which are presented in Table $5 a, b, c$. 
Table 5

Chemical composition of initial samples of the Saryjaz area black shale formation

\begin{tabular}{|c|c|c|c|c|c|c|c|c|c|c|c|c|c|c|c|}
\hline \multicolumn{16}{|c|}{$a$} \\
\hline 变 & $\mid \begin{array}{l}\mathbf{s} \\
\text { upó6 }\end{array}$ & Mn & $\mathrm{Ni}$ & $\mathrm{C}_{0}$ & $\mathbf{T i}$ & $\mathrm{v}$ & $\mathrm{Cr}$ & Mo & w & $\mathrm{Zr}$ & $\mathrm{Nb}$ & In & $\mathrm{Cu}$ & $\mathrm{Pb}$ & $\mathrm{Ag}$ \\
\hline & & $10-2$ & $10-3$ & 10-3 & $10-1$ & 10-2 & $10-3$ & 10-3 & $10-2$ & $10-2$ & $10-3$ & $10-3$ & $10-3$ & 10-3 & 10- 4 \\
\hline प-Сस & $1(\mathrm{k})$ & - & 4 & - & 4 & 15 & 5 & 12 & - & 0,5 & - & - & 9 & 12 & 3 \\
\hline \begin{tabular}{|l|}
$\mathrm{M}$ \\
$\mathrm{i} / \mathrm{in}$
\end{tabular} & \begin{tabular}{|l|}
$\begin{array}{l}\mathrm{Nk} \\
\text { mpo }\end{array}$ \\
\end{tabular} & $\mathrm{Sb}$ & $\mathrm{Bi}$ & As & $\mathrm{Zn}$ & Cd & Sn & $\mathrm{Ge}$ & $\mathrm{Ga}$ & $\mathrm{Yb}$ & $\mathbf{Y}$ & $\mathrm{La}$ & $\mathbf{P}$ & $\mathrm{Be}$ & $\mathrm{Sr}$ \\
\hline & & $10-2$ & 10-3 & $10-2$ & $10-2$ & $10-2$ & $10-3$ & $10-3$ & $10-3$ & $10-3$ & $10-3$ & $10-2$ & $10-1$ & 10- 4 & 10-2 \\
\hline ч.СЖ & $1(\mathrm{k})$ & - & - & 5 & 0,3 & - & 0,15 & - & 0,5 & 0,3 & 3 & - & - & - & 2 \\
\hline 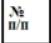 & \begin{tabular}{|l|}
$\begin{array}{l}\text { se } \\
\text { mpoб }\end{array}$ \\
\end{tabular} & $\mathrm{Ba}$ & Li & $\mathrm{Ta}$ & Th & $\mathbf{U}$ & Au & $\mathrm{Sc}$ & $\mathrm{SiO}_{2}$ & $\mathrm{AL}_{2} \mathrm{O}_{3}$ & $\mathrm{MgO}$ & $\mathrm{Fe}_{2} \mathrm{O}_{3}$ & $\mathrm{CaO}$ & $\mathrm{Na}_{2} \mathrm{O}$ & $\mathrm{K}_{2} \mathrm{O}$ \\
\hline & & $10-2$ & 10- 3 & 10. 1 & 10-2 & 10- 1 & $10-3$ & $10-3$ & $\%$ & $\%$ & $\%$ & $\%$ & $\%$ & $\%$ & $\%$ \\
\hline ч.Сж & $1(\mathrm{k})$ & - & - & - & - & - & - & - & 70 & 3 & 2 & 3 & 1,2 & - & - \\
\hline
\end{tabular}

There is still no consensus in the literature on the forms of finding noble and rare metals, on their effective methods for determining their actual concentration in iron oxides and hydroxides, clay minerals, micas, chlorites, alunite, quartz-chalcedony, jarosite, some other carrier minerals, as well as in carbon-bitumen inclusions in shales. The black-shale ores of the studied objects belong to the category of highly resistant and require special technological approaches.

\begin{tabular}{|c|c|c|c|c|c|c|c|c|c|c|c|c|c|c|c|}
\hline \begin{tabular}{|l|}
$x 6$ \\
$\pi / 4$ \\
$\pi / 4$
\end{tabular} & \begin{tabular}{|l|} 
Nó \\
mpoô
\end{tabular} & Mn & $\mathbf{N i}$ & $\mathrm{Co}$ & $\mathbf{T i}$ & V & $\mathrm{Cr}$ & Mo & W & $\mathrm{Zr}$ & $\mathrm{Nb}$ & In & $\mathrm{Cu}$ & $\mathbf{P b}$ & $\mathrm{Ag}$ \\
\hline & & $10-2$ & $10-3$ & $10-3$ & $10-1$ & 10-2 & $10-3$ & 10-3 & $10-2$ & 10-2 & 10-3 & $10-3$ & $10-3$ & $10-3$ & 10- 4 \\
\hline प-Cस & 2(k) & 12 & 15 & - & 4 & 30 & 15 & 15 & - & 2 & - & - & 40 & 15 & 5 \\
\hline \multirow[t]{2}{*}{ 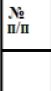 } & 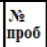 & $\mathrm{Sb}$ & $\mathrm{Bi}$ & As & $\mathrm{Zn}$ & $\mathrm{Cd}$ & Sn & $\mathrm{Ge}$ & $\mathrm{Ga}$ & $\mathrm{Yb}$ & $\mathbf{Y}$ & $\mathrm{La}$ & $\mathbf{P}$ & $\mathrm{Be}$ & $\mathrm{Sr}$ \\
\hline & & $10-2$ & $10-3$ & $10-2$ & $10-2$ & $10-2$ & 10-3 & $10-3$ & 10-3 & $10-3$ & $10-3$ & $10-2$ & $10-1$ & 10- 4 & $10-2$ \\
\hline Ч-Сस & $2(\mathrm{k})$ & - & - & 12 & 1,2 & - & 0,15 & - & 0,5 & 0,3 & 3 & - & - & - & 2 \\
\hline \multirow[t]{2}{*}{ 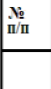 } & \begin{tabular}{|l|} 
Nó \\
mp0б
\end{tabular} & $\mathrm{Ba}$ & $\mathbf{L i}$ & $\mathrm{Ta}$ & Th & $\mathbf{U}$ & $\mathrm{Au}$ & $\mathrm{Sc}$ & $\mathrm{SiO}_{2}$ & $\mathrm{AL}_{2} \mathrm{O}_{3}$ & Mg0 & $\mathrm{Fe}_{2} \mathrm{O}_{3} \mid$ & $\mathrm{CaO}$ & $\mathrm{Na}_{2} \mathrm{O}$ & $\mathrm{K}_{2} \mathrm{O}$ \\
\hline & & $10-2$ & 10- 3 & $10-1$ & $10-2$ & $10-1$ & $10-3$ & $10-3$ & $\%$ & $\%$ & $\%$ & $\%$ & $\%$ & $\%$ & $\%$ \\
\hline Ч-Сस & $2(\mathrm{k})$ & 3 & - & - & - & - & - & - & 70 & 7 & 4 & 5 & 4 & 0,7 & 0,2 \\
\hline
\end{tabular}




\begin{tabular}{|c|c|c|c|c|c|c|c|c|c|c|c|c|c|c|c|}
\hline $\begin{array}{l}\mathrm{No} \\
\mathrm{n} / \mathrm{I}\end{array}$ & \begin{tabular}{l|} 
№ \\
Ipроб
\end{tabular} & Mn & $\mathrm{Ni}$ & $\mathrm{C}_{0}$ & $\mathrm{Ti}$ & $\mathrm{V}$ & $\mathrm{Cr}$ & Mo & W & $\mathrm{Zr}$ & $\mathrm{Nb}$ & In & $\mathrm{Cu}$ & $\mathrm{Pb}$ & $\mathrm{Ag}$ \\
\hline & & $10-2$ & $10-3$ & $10-3$ & $10-1$ & $10-2$ & $10-3$ & $10-3$ & $10-2$ & 10-2 & 10-3 & $10-3$ & $10-3$ & 10-3 & 10- 4 \\
\hline Ч.СЖ & $2(\mathrm{~K})$ & 12 & 15 & - & 4 & 30 & 15 & 15 & - & 2 & - & - & 40 & 15 & 5 \\
\hline \multirow[t]{2}{*}{$\begin{array}{l}\mathrm{No} \\
\mathrm{N} / \mathrm{m}\end{array}$} & \begin{tabular}{|l|}
\multirow{2}{*}{} \\
проб
\end{tabular} & $\mathrm{Sb}$ & $\mathrm{Bi}$ & As & $\mathrm{Zn}$ & $\mathrm{Cd}$ & Sn & $\mathrm{Ge}$ & $\mathrm{Ga}$ & $\mathrm{Yb}$ & $\mathbf{Y}$ & $\mathrm{La}$ & $P$ & $\mathrm{Be}$ & $\mathrm{Sr}$ \\
\hline & & $10-2$ & $10-3$ & 10-2 & $10-2$ & $10-2$ & 10- 3 & $10-3$ & $10-3$ & $10-3$ & $10-3$ & 10-2 & $10-1$ & 10- 4 & $10-2$ \\
\hline Ч.СЖ & $2(\mathrm{~K})$ & - & - & 12 & 1,2 & - & 0,15 & - & 0,5 & 0,3 & 3 & - & - & - & 2 \\
\hline \multirow[t]{2}{*}{ 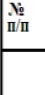 } & $\begin{array}{l}\mathbf{N} \mathbf{2} \\
\text { appố }\end{array}$ & $\mathrm{Ba}$ & $\mathbf{L i}$ & $\mathrm{Ta}$ & Th & $\mathbf{U}$ & Au & $\mathrm{Sc}$ & $\mathrm{SiO}_{2}$ & $\mathrm{AL}_{2} \mathrm{O}_{3}$ & Mg0 & $\mathrm{Fe}_{2} \mathrm{O}_{3}$ & $\mathrm{CaO}$ & $\mathrm{Na}_{2} \mathrm{O}$ & $\mathrm{K}_{2} \mathrm{O}$ \\
\hline & & $10-2$ & $10-3$ & 10- 1 & $10-2$ & $10-1$ & 10-3 & $10-3$ & $\%$ & $\%$ & $\%$ & $\%$ & $\%$ & $\%$ & $\%$ \\
\hline ч-Сж & $2(\mathrm{~K})$ & 3 & - & - & - & - & - & - & 70 & 7 & 4 & 5 & 4 & 0,7 & 0,2 \\
\hline
\end{tabular}

Then the ore-bearing rocks of the black shale formation crushed to the specified value were further crushed in a non-standard crushing unit with a three-phase electric motor $3000 \mathrm{rpm} 2 \mathrm{~kW}$, at a pressure of 30-60 atm/ $/ \mathrm{cm}^{2}$.

Non-standard crushing unit consists of a cylindrical cup and a lid, so that during crushing no dust is released into the environment the cup is tightly closed with a lid. The cup and the lid are made of ceramic. Crushing the loaded sample of 20 grams lasts for 30-60 minutes. At the same time the dust of the crushed mineral does not settle in water, this is one of the visual indications that, the size of the crushed particles is less than a micron.

The size and morphological structure of the dispersed particles were studied using a scanning electron microscope (SEM) [8,9] Fig. 2. 
$a$



$c$

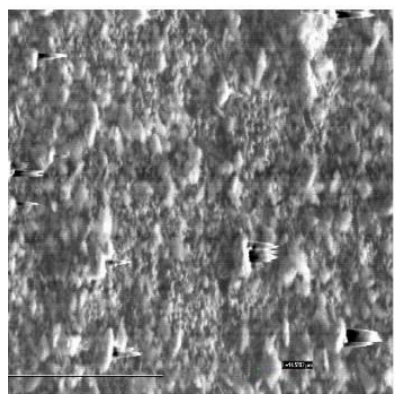

Magnification-E $x 1010 ; L=14.5782 \mu \mathrm{m}$

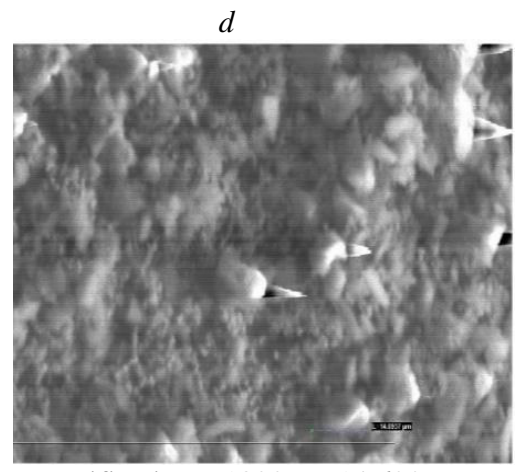

Magnification $x 1800 ; L=14.6937$

$\mu \mathrm{m}$

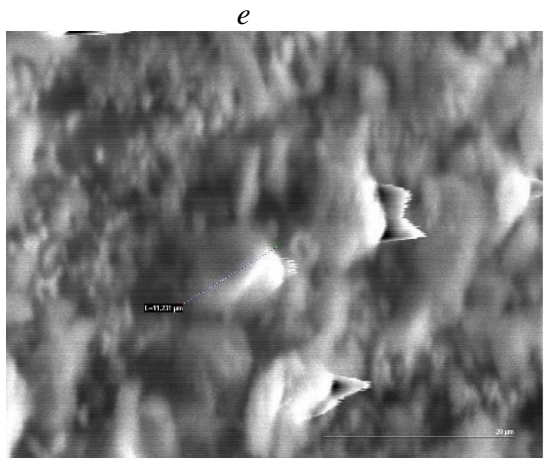

Magnification $x$ 4000; $L=11.231 \mu \mathrm{m}$

Fig. 2. Morphological structure of dispersed particles

From figure $2(a)(L=15.0537 \mu \mathrm{m})$ and $(b)(L=16.4966 \mu \mathrm{m})$ the mass uniformity and regularity of the mineral structure is observed, while figures $(c)(L=14.5782 \mu \mathrm{m}), \quad(d) \quad(L=14.6937 \mu \mathrm{m}), \quad(e)$ $(L=11.231 \mu \mathrm{m})$ suggest a gritty and slightly clastic structure.

In publications of scientists in the field of geology and mining, "black-shales" are considered as a new promising and unconventional source of noble and rare-metal raw materials.

Analysis of studies and publications confirms that black-shale ores of the studied objects belong to the category of highly refractory and require special technological approaches. 
During the fragmentation of the Saryjaz area black shale formation there are changes in the quantitative content of their constituent components, as evidenced by the obtained data on the spectral analysis of Table 6, 7, 8 .

As can be seen from Table 6, an increase in the quantitative content of metals $(\mathrm{g} / \mathrm{t})$ in the samples of coarse fraction milling from the first point coordinate BSSF 1(b) (g/t) Ni , Ti (from 3-4); Mo (from 212); $\mathrm{Cu}$ (from 5-9); $\mathrm{Pb}$ (from 1.5-12; Ag (from 1.5-3); such metals as $\mathrm{As}, \mathrm{Zn}, \mathrm{Sn}, \mathrm{Ga}$ begin to appear and the quantitative content of $\mathrm{V}$ (from 40-15); Cr (from 40-5); $\mathrm{Zr}$ (from 5-1.5); Yb (from 0.7-9.3) and Y (from 7-3) decreases;

The quantitative content of metals in coarse fraction samples from the second point coordinate BSSF 2 (b) (g/t) Ni (from 3-15); Ti (from 3-4); $\mathrm{Mo}$ (from 2-15); $\mathrm{Cu}$ (from 5-40); $\mathrm{Pb}$ (from 1.5-15) also increases: $\mathrm{Ag}$ (from 1.5-5); at the same time, there is a manifestation of metals $\mathrm{Mn}, \mathrm{As}, \mathrm{Zn}, \mathrm{Sn}, \mathrm{Ga}$ and a reduction of some metals as $\mathrm{V}$ (from 40-30); $\mathrm{Cr}$ (from 40-15); $\mathrm{Zr}$ (from 5-2); $\mathrm{Yb}$ (from 0.7-0.3); Y (from 7-3); $\mathrm{Sr}$ (from 3-2).

In samples from the third point coordinate BSSF 3(b) observed increase in the quantitative content of metals - Mo (from 2-20); $\mathrm{Cu}$ (from 5-7); $\mathrm{Pb}$ (from 1.5-12); $\mathrm{Ag}$ (from 1.5-4); remains unchanged $\mathrm{Ni}$ (3-3) and Ti (3-3); As, Zn, Sn, Ga and decreases of Ti (from 3-2); $\mathrm{V}$ (from 40-30); Cr (from 40-5); $\mathrm{Zr}$ (from 5-1,5); Yb (from 0,7-0,3) and $\mathrm{Y}$ (from 7-3).

Table 6 Comparative characteristics of the chemical composition of the fragmented samples of the black shale formation from the first coordinate point of BSSF $1(b)$ $L=16.4966 \mu \mathrm{m}$; BSSF $1(\mathrm{~m}) L=14.5782 \mu \mathrm{m}$; BSSF 1 (s) $L=11.231 \mu \mathrm{m}$; with initial data

\begin{tabular}{l|l|l|l|l|l|l|l|l|l}
\hline \multirow{2}{*}{ №o samples } & $\mathrm{Mn}$ & $\mathrm{Ni}$ & $\mathrm{Co}$ & $\mathrm{Ti}$ & $\mathrm{V}$ & $\mathrm{Cr}$ & $\mathrm{Mo}$ & $\mathrm{Zr}$ & $\mathrm{Cu}$ \\
\cline { 2 - 10 } & $10-2$ & $10-3$ & $10-3$ & $10-1$ & $10-2$ & $10-3$ & $10-3$ & $10-2$ & $10-3$ \\
\hline $\begin{array}{l}\text { Original } \\
\text { sample }\end{array}$ & & 3 & & 3 & 40 & 40 & 2 & 5 & 5 \\
\hline BSSF1b & & 4 & & 4 & 15 & 5 & 12 & 0,5 & 9 \\
\hline BSSF1m & 5 & 15 & 0,3 & 5 & 20 & 15 & 30 & 2 & 50 \\
\hline BSSF1s & 3 & 12 & 0,3 & 4 & 12 & 15 & 30 & 1,5 & 200 \\
\hline $\begin{array}{l}\text { №№ sam- } \\
\text { ples }\end{array}$ & $\mathrm{Pb}$ & $\mathrm{Ag}$ & $\mathrm{As}$ & $\mathrm{Zn}$ & $\mathrm{Sn}$ & $\mathrm{Ga}$ & $\mathrm{Yb}$ & $\mathrm{Y}$ & $\mathrm{Sr}$ \\
\cline { 2 - 10 } & $10-3$ & $10-4$ & $10-2$ & $10-2$ & $10-3$ & $10-3$ & $10-3$ & $10-3$ & $10-2$ \\
\hline $\begin{array}{l}\text { Original } \\
\text { sample. }\end{array}$ & 1,5 & 1,5 & - & - & - & - & 0,7 & 7 & 3 \\
\hline
\end{tabular}




\begin{tabular}{l|l|l|l|l|l|l|l|l|l}
\hline BSSF1b & 12 & 3 & 5 & 0,3 & 0,15 & 0,5 & 0,3 & 3 & 2 \\
\hline BSSF1m & 20 & 3 & 20 & 0,7 & 0,15 & 0,7 & 0,3 & 3 & 2 \\
\hline BSSF1s & 30 & 3 & 15 & 2 & 0,15 & 0,5 & 0,3 & 3 & 2 \\
\hline $\begin{array}{l}\text { № } \\
\text { № samples }\end{array}$ & & $\mathrm{Ba}$ & $\mathrm{SiO}_{2}$ & $\mathrm{Al}_{2} \mathrm{O}_{3}$ & $\mathrm{MgO}$ & $\mathrm{Fe}_{2} \mathrm{O}_{3}$ & $\mathrm{CaO}$ & $\mathrm{Na}_{2} \mathrm{O}$ & $\mathrm{R}_{2} \mathrm{O}$ \\
\hline $\begin{array}{l}\text { Original } \\
\text { sample. }\end{array}$ & & - & 70 & 5 & 2 & 2 & 0,5 & - & - \\
\hline BSSF1b & & - & 70 & 3 & 2 & 3 & 1,2 & - & - \\
\hline BSSF1m & & 2 & 70 & 5 & 4 & 9 & 2 & 0,2 & 0,1 \\
\hline BSSF1s & & 2 & 70 & 4 & 3 & 7 & 1,5 & 0,15 & - \\
\hline
\end{tabular}

Table 7

Comparative characteristics of the chemical composition of the fragmented samples of the black shale formation from the second coordinate point of BSSF $2(b)$ $L=16.4966 \mu \mathrm{m}$; BSSF $2(\mathrm{~m}) L=14.5782 \mu \mathrm{m}$; BSSF $2(s) L=11.231 \mu \mathrm{m}$; with initial data

\begin{tabular}{|c|c|c|c|c|c|c|c|c|c|}
\hline \multirow{2}{*}{$\begin{array}{l}\text { № } \\
\text { № samples }\end{array}$} & $\mathrm{Mn}$ & $\mathrm{Ni}$ & Co & $\mathrm{Ti}$ & $\mathrm{V}$ & $\mathrm{Cr}$ & Mo & $\mathrm{Zr}$ & $\mathrm{Cu}$ \\
\hline & $10-2$ & $10-3$ & $10-3$ & $10-1$ & $10-2$ & $10-3$ & $10-3$ & $10-2$ & $10-3$ \\
\hline $\begin{array}{l}\text { Original } \\
\text { sample. }\end{array}$ & & 3 & & 3 & 40 & 40 & 2 & 5 & 5 \\
\hline BSSF 2b & 12 & 15 & & 4 & 30 & 15 & 15 & 2 & 40 \\
\hline BSSF $2 \mathrm{~m}$ & 5 & 20 & & 4 & 5 & 12 & 30 & 3 & 300 \\
\hline BSSF2s & 2 & 15 & 0,3 & 3 & 15 & 12 & 15 & 2 & 300 \\
\hline \multirow[t]{2}{*}{ № samples } & $\mathrm{Pb}$ & $\mathrm{Ag}$ & As & $\mathrm{Zn}$ & $\mathrm{Sn}$ & $\mathrm{Ga}$ & $\mathrm{Yb}$ & $\mathrm{Y}$ & $\mathrm{Sr}$ \\
\hline & $10-3$ & $10-4$ & $10-2$ & $10-2$ & $10-3$ & $10-3$ & $10-3$ & $10-3$ & $10-2$ \\
\hline $\begin{array}{l}\text { Original } \\
\text { sample. }\end{array}$ & 1,5 & 1,5 & - & - & - & - & 0,7 & 7 & 3 \\
\hline BSSF2b & 15 & 5 & 12 & 1,2 & 0,15 & 0,5 & 0,3 & 3 & 2 \\
\hline BSSF2m & 30 & 9 & 15 & 1,2 & 0,15 & 0,5 & 0,3 & 3 & 2 \\
\hline BSSF2s & 20 & 4 & 9 & 1,5 & 0,15 & 0,5 & 0,3 & 3 & 2 \\
\hline \multirow{2}{*}{$\begin{array}{l}\text { № } \\
\text { № samples }\end{array}$} & & $\mathrm{Ba}$ & $\mathrm{SiO}_{2}$ & $\mathrm{Al}_{2} \mathrm{O}_{3}$ & $\mathrm{MgO}$ & $\mathrm{Fe}_{2} \mathrm{O}_{3}$ & $\mathrm{CaO}$ & $\mathrm{Na}_{2} \mathrm{O}$ & $\mathrm{R}_{2} \mathrm{O}$ \\
\hline & & $10-2$ & $\%$ & $\%$ & $\%$ & $\%$ & $\%$ & $\%$ & $\%$ \\
\hline $\begin{array}{l}\text { Original } \\
\text { sample. }\end{array}$ & & - & 70 & 5 & 2 & 2 & 0,5 & - & - \\
\hline BSSF2b & & 3 & 70 & 7 & 4 & 5 & 4 & 0,7 & 0,2 \\
\hline BSSF2m & & 3 & 70 & 9 & 4 & 7 & 5 & 0,9 & 0,1 \\
\hline BSSF2s & & - & 70 & 3 & 2 & 7 & 2 & 0,15 & - \\
\hline
\end{tabular}


Table 8

Comparative characteristics of the chemical composition of the fragmented samples of the black shale formation from the third coordinate point BSSF $3(b) L=16.4966$ $\mu \mathrm{m}$; BSSF $3(m) L=14.5782 \mu \mathrm{m}$; BSSF 3 (s) $L=11.231 \mu \mathrm{m}$; with initial data

\begin{tabular}{l|l|l|l|l|l|l|l|l|l}
\hline $\begin{array}{l}\text { № } \\
\text { № sam- } \\
\text { ples }\end{array}$ & $\mathrm{Mn}$ & $\mathrm{Ni}$ & $\mathrm{Co}$ & $\mathrm{Ti}$ & $\mathrm{V}$ & $\mathrm{Cr}$ & $\mathrm{Mo}$ & $\mathrm{Zr}$ & $\mathrm{Cu}$ \\
\cline { 2 - 10 } & $10-2$ & $10-3$ & $10-3$ & $10-1$ & $10-2$ & $10-3$ & $10-3$ & $10-2$ & $10-3$ \\
\hline $\begin{array}{l}\text { Original } \\
\text { sample }\end{array}$ & - & 3 & - & 3 & 40 & 40 & 2 & 5 & 5 \\
\hline BSSF3b & - & 3 & - & 2 & 30 & 5 & 20 & 1,5 & 7 \\
\hline BSSF3m & 0,5 & 1,2 & - & 3 & 20 & 2 & 5 & 1,2 & 5 \\
\hline BSSF3s & 15 & 15 & 0,3 & 4 & 15 & 20 & 20 & 5 & 500 \\
\hline $\begin{array}{l}\text { №№ } \\
\text { samples }\end{array}$ & $\mathrm{Pb}$ & $\mathrm{Ag}$ & $\mathrm{As}$ & $\mathrm{Zn}$ & $\mathrm{Sn}$ & $\mathrm{Ga}$ & $\mathrm{Yb}$ & $\mathrm{Y}$ & $\mathrm{Sr}$ \\
\hline & $10-3$ & $10-4$ & $10-2$ & $10-2$ & $10-3$ & $10-3$ & $10-3$ & $10-3$ & $10-2$ \\
\hline $\begin{array}{l}\text { Original } \\
\text { sample }\end{array}$ & 1,5 & 1,5 & - & - & - & - & 0,7 & 7 & 3 \\
\hline BSSF3b & 12 & 4 & 7 & 0,5 & 0,15 & 0,5 & 0,3 & 3 & 2 \\
\hline BSSF3m & 7 & 2 & 9 & 1,5 & 0,15 & 0,5 & 0,3 & 3 & 2 \\
\hline BSSF3s & 30 & 5 & 30 & 5 & 0,15 & 0,5 & 0,3 & 3 & 2 \\
\hline $\begin{array}{l}\text { №№ } \\
\text { samples }\end{array}$ & & $\mathrm{Ba}$ & $\mathrm{SiO}_{2}$ & $\mathrm{Al}_{2} \mathrm{O}_{3}$ & $\mathrm{MgO}$ & $\mathrm{Fe} 2 \mathrm{O}_{3}$ & $\mathrm{CaO}$ & $\mathrm{Na} 2 \mathrm{O}$ & $\mathrm{R}_{2} \mathrm{O}$ \\
\hline & & $10-2$ & $\%$ & $\%$ & $\%$ & $\%$ & $\%$ & $\%$ & $\%$ \\
\hline $\begin{array}{l}\text { Original } \\
\text { sample }\end{array}$ & & - & 70 & 5 & 2 & 2 & 0,5 & - & - \\
\hline BSSF3b & & - & 70 & 2 & 1,5 & 3 & 0,5 & - & - \\
\hline BSSF3m & & 2 & 50 & 2 & 1,2 & 1,5 & 0,4 & - & - \\
\hline BSSF3s & & - & 70 & 4 & 3 & 7 & 2 & 0,15 & - \\
\hline
\end{tabular}

Note:(b)-big; (m)-medium; $(s)$-small fract

According to the results of spectral analysis in Table 7, the same picture is observed: Increase in the amount of metals in the average fraction of the milled material from the first coordinate point $(\mathrm{g} / \mathrm{t}) \mathrm{BSSF} 1(\mathrm{~m}) \mathrm{Ni}$ (from 3-15); Ti(from 3-5); Mo (from 2-30); $\mathrm{Cu}$ (from 5-50); $\mathrm{Pb}$ (from 1.5-20); Ag (from 1.5-3); the metals Mn, Co, As, Zn, Sn, Ga, Ba decrease in quantitative content V (from 40-20); Cr (from 40-15); Zn (from 5-2); $\mathrm{Yb}$ (from 0.7-0.3); Y (from 7-3); Sr (from 3-2).

In the samples of the middle fraction of the crushed material from the second point of coordinate BSSF 2 (m) there is an increase in the quantitative content of metals $\mathrm{Ni}$ (from 3-20); Ti (from 3-4); Mo (from 2-30); $\mathrm{Cu}$ (from 5-300); Pb (from 1,5-30); Ag (from 1,5 -9); Mn, As, Zn, Sn, Ga, Ba manifestations and decrease of metals $\mathrm{V}$ (from 40-5); $\mathrm{Cr}$ (from 40-5); $\mathrm{Zr}$ (from 5-3); Yb (from 0,7-0,3); Y (from 7-3); Sr (from 3-2). 
In the middle fractions of the third point, the coordinate of the BSSF 3 (m) remains unchanged $\mathrm{Ti}(3-3)$; $\mathrm{Cu}(5-5)$; $\mathrm{Sr}$ (2-2); the quantitative content of metals $\mathrm{Mo}$ (from 2-5); $\mathrm{Pb}$ (from 1.5-7); $\mathrm{Ag}$ (from 1.5-2) increases; $\mathrm{Mn}, \mathrm{As}, \mathrm{Zn}, \mathrm{Sn}, \mathrm{Ga}, \mathrm{Ba}$ appear; and there is a decrease in the quantitative content of $\mathrm{Ni}$ (from 3-1.2), V (from 40-20); Cr (from 40-2); $\mathrm{Zr}$ (from 5-1. 2); $\mathrm{Yb}$ (from 0.7-0.3); Y (from 7-3) and $\mathrm{Sr}$ (3-2).

Table 8 shows the data of the spectral analysis of small fractions the coordinates of points BSSF1 (s), BSSF 2 (s) and BSSF 3 (s) where an increase of quantitative metal content by

BSSF 1 (s) Ni (from 3-12); Ti (from 3-4); Mo (from 2-30); $\mathrm{Cu}$ (from 5-200); $\mathrm{Pb}$ (from 1.5-30); $\mathrm{Ag}$ (from 1.5-3);

In BSSF 2 (s) Ni (from 3-15); Mo (from 2-15); Cu (from 5-300); $\mathrm{Pb}$ (from 1.5-20); Ag (from 1.5-4);

By BSSF 3 (s) Ni (from 3-15); Ti (from 3-4); Mo (from 2-20); Cu (from 5-500); $\mathrm{Pb}$ (from 1.5-30); $\mathrm{Ag}$ (from 1.5-5); $\mathrm{Zr}$ (5-5) remains unchanged.

Metals are revealed by BSSF 1 (s) Mn, Co, As, Zn, Sn, Ga, Ba; BSSF 2 (s) Mn, Co, As, Zn, Sn, Ga, Ba; BSSF 3 (s) Mn, Co, As, Zn, $\mathrm{Sn}, \mathrm{Ga}, \mathrm{Ba}$; Metals in samples BSSF 1 (s) V (from 40-12) are decreased; $\mathrm{Cr}$ (from 40-15); Yb (from 0.7-0.3); Y (from 7-3); $\mathrm{Sr}$ (from 32); BSSF 2(s) V (from 40-15); Cr (from 40-12); Zr (from 5-2); Yb (from 0.7-0.3); Y (from 7-3); Sr (from 3-2); BSSF 3(s) V (from 40-15); Cr (from 40-20); Yb (from 0.7-0.3); Y (from 7-3); Sr (from 3-2

The quantitative increase of metals in the samples is shown in Figures 3,4,5.

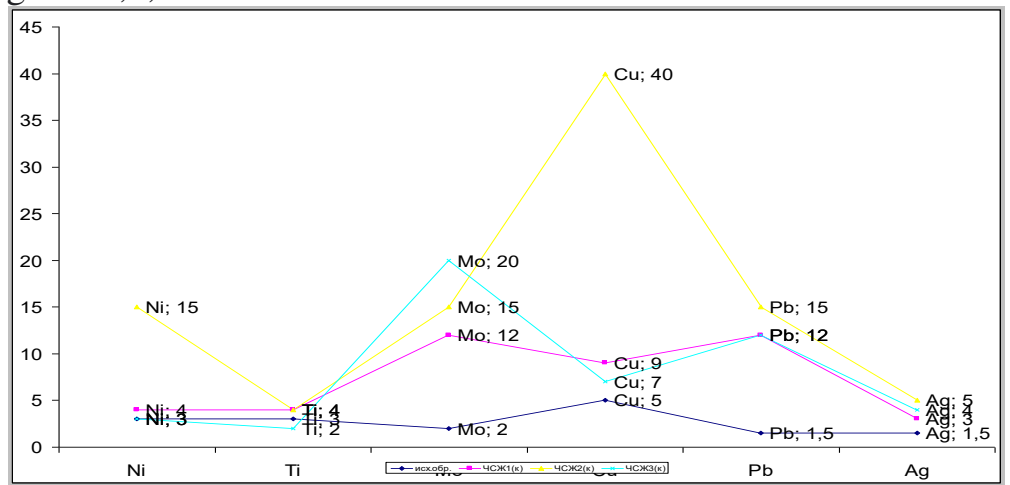

Fig. 3. Change in the quantitative composition of minerals during fragmentation to (b) $(L=15.0537 \mu \mathrm{m})$ and $(L=16.4966 \mu \mathrm{m})$ 


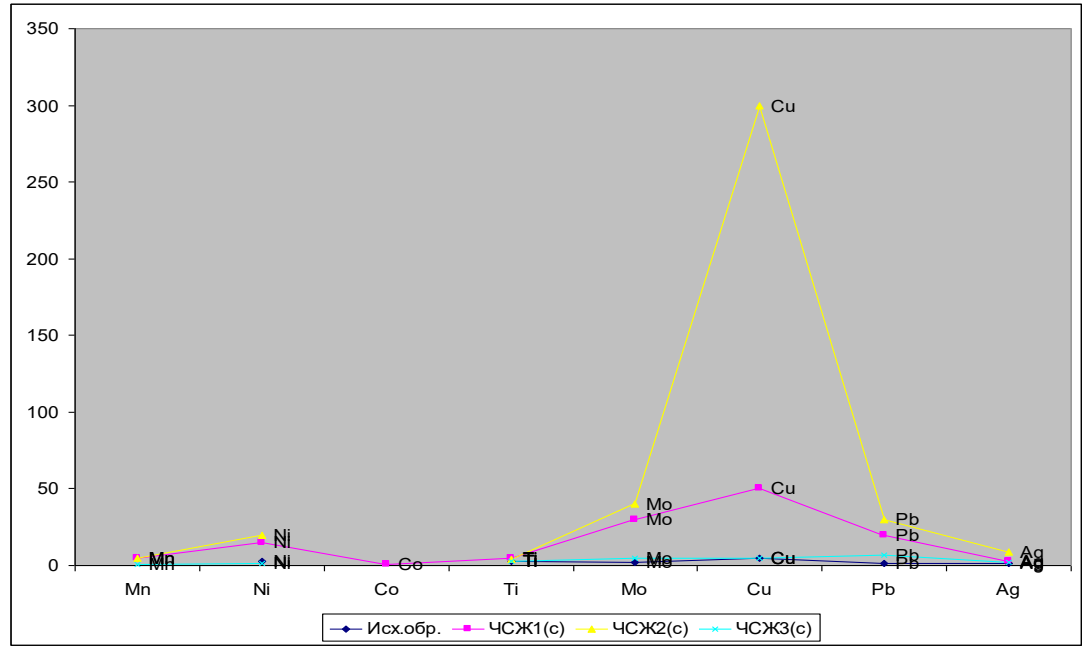

Fig. 4. Change in the quantitative composition of minerals during fragmentation to (m) $(L=14.5782 \mu \mathrm{m}),(L=14.6937 \mu \mathrm{m})$

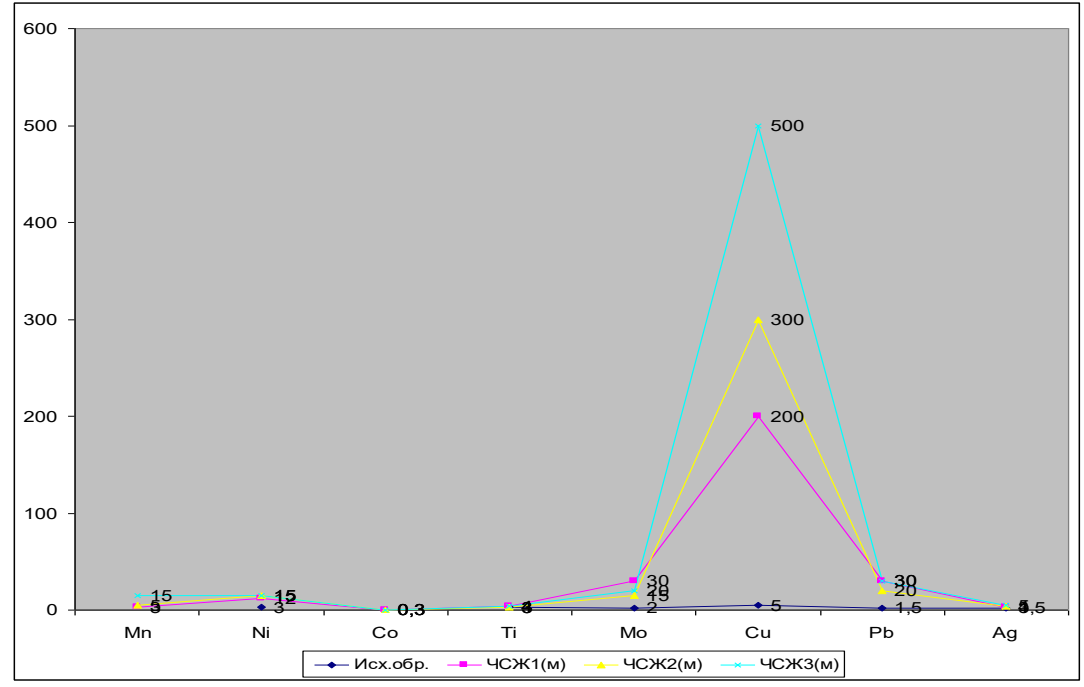

Fig. 5. Change in the quantitative composition of minerals during fragmentation to (s) $(L=11.231 \mu \mathrm{m})$

As can be seen from the table and figures, when the samples were broken down to chunk size $(b)(L=15.0537 \mu \mathrm{m}),(L=16.4966 \mu \mathrm{m})$; 
medium particle size $(m)(L=14.5782 \mu \mathrm{m}),(L=14.6937 \mu \mathrm{m})$ and in the smallest particles $(s)(L=11.231$ micrometers $)$ is observed a presence of some metals $\mathrm{Mn}, \mathrm{Co}, \mathrm{As}, \mathrm{Zn}, \mathrm{Sn}, \mathrm{Ga}$, Ba which were not detected in the original sample; also is observed an increase of the quantitative content of some metals in all fractured samples in comparison with the original sample, especially in the smallest sample $\mathrm{BSSF} 1(s) \mathrm{Ni}$ (from 3-12); Ti (from 3-4); Mo (from 2-30); $\mathrm{Cu}$ (from 5-200); Pb (from1,5-30); Ag (from 1,5-3);

In BSSF 2 (s) Ni (from 3-15); Mo (from 2-15); Cu (from 5-300); $\mathrm{Pb}$ (from 1.5-20); Ag (from 1.5-4);

In BSSF 3 (s) Ni (from 3-15); Ti (from 3-4); Mo (from 2-20); $\mathrm{Cu}$ (from 5-500); $\mathrm{Pb}$ (from1.5-30); $\mathrm{Ag}$ (from 1.5-5); $\mathrm{Ni}$ (from 3-15); $\mathrm{Mo}$ (from 2-20); $\mathrm{Pb}$ (from 120); $\mathrm{Ag}$ (from 120); $\mathrm{Ni}$ (from 400); $\mathrm{Ni}$ (from 110 ); $\mathrm{Ni}$ (from 110); $\mathrm{Ni}$ (from 110); $\mathrm{Pb}$ (from 110). (from 1.5-5);

\subsection{Technological scheme of enrichment of valuable components of the black shale formation}

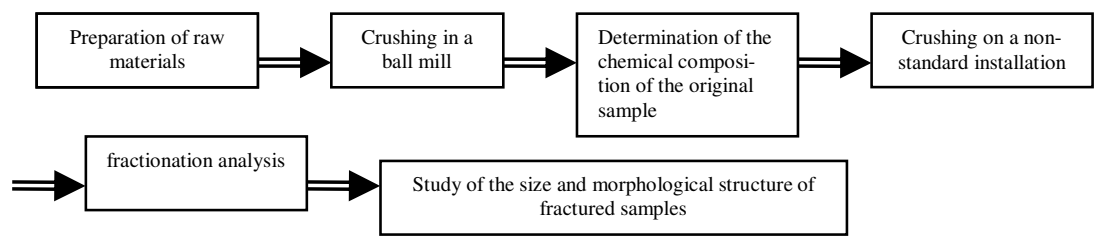

\section{Conclusions}

1. Chemical composition and technological properties of initial samples were studied;

2. Spectral analysis of initial and split samples was carried out;

3 . When the samples were crushed to the size of lumps $(b)$ $(L=15.0537 \mu \mathrm{m}), \quad(L=16.4966 \mu \mathrm{m})$; medium particle size $(\mathrm{m})$ $(L=14.5782 \mu \mathrm{m}),(L=14.6937 \mu \mathrm{m}) ;$ and to the small particles $(\mathrm{s})$ 
$(L=11.231 \mu \mathrm{m})$ is observed manifestation of some metals $\mathrm{Mn}, \mathrm{Co}$, As, $\mathrm{Zn}, \mathrm{Sn}, \mathrm{Ga}, \mathrm{Ba}$ which were not known in initial samples; increase of quantitative content of some metals is observed in all crushed samples in comparison with initial sample, especially increase of quantitative content of metals is observed in melkozrushed sample BSSF 1(s), BSSF 2 (s), BSSF 3 (s) Ni (from 3-15); Ti (from 3-4); $\mathrm{Mo}$ (from 2-30); $\mathrm{Cu}$ (from 5-500); $\mathrm{Pb}$ (from1,5-30); $\mathrm{Ag}$ (from 1,5-5);

4. Thus, to increase the quantitative content of the above metals Optimal size of dispersed particles is $L=11.231 \mu \mathrm{m}$.

\section{Literature}

1. http://www.miningexpo.ru/3672).

2. Patent for invention No. 2455237. Nanotechnological method for extracting rhenium from rocks and ores of black shale formations and products of their processing. (Date of application: 06.12.2010) 10.07.2012.

3. Shumilova L.V. Combined methods of cuvette and heap leaching of refractory gold-bearing raw materials based on directed photoelectrochemical effects. Abstract of the dissertation for the degree of Doctor of Technical Sciences. Chita, 2010.- http://textarchive.ru/c-1318156-pall.html-12.06.17.

4. Special and combined methods of mineral processing: textbook / V. G. Samoilik - Donetsk: OOO "Skhidniy vydavnichiy dim", 2015.- p. 108.

5. Vorobyov N.I., Novik D.M. Enrichment of minerals (textbook). Minsk: BSTU, 2008.-174p.

6. Adamov E.V. Technology of non-ferrous metal ores. M, 2001.-470 s.

7. GOST 30439 (96), Pesticides, Sieve analysis, OKS: 65100 KGS: L19, Test methods.

8. Scanning electron microscope. Study guide for students of the Faculty of Physics and Technology. / Compiled by: V.P. Makarov, O.N. Kanygina KyrgyzRussian Slavic University.-Bishkek. 2006.25 p.

9. Goldstein, J. Scanning electron microscopy and X-ray microanalysis: in 2 volumes / J. Goldstein, D. Newbury, P. Echlin et al. - M .: Mir, 1984. 